\title{
Descrição do movimento da articulação metacarpofalangiana de equinos pela metodologia baseada em videogrametria
}

[Equine metacarpophalangeal joint movement using videogrammetry method]

\author{
R.M. Albernaz ${ }^{1}$, R.C. Basile ${ }^{1}$, D.P.M. Dias ${ }^{1}$, A.O. Paiva-Neto ${ }^{1}$, L.M.W. Gomide ${ }^{1}$ \\ A. Queiroz-Neto ${ }^{2}$, J.C. Lacerda-Neto ${ }^{1}$ \\ ${ }^{1}$ Departamento de Clínica e Cirurgia Veterinária - FCAV-UNESP - Jaboticabal, SP \\ ${ }^{2}$ Departamento de Morfologia e Fisiologia Animal - FCAV-UNESP - Jaboticabal, SP
}

\begin{abstract}
RESUMO
Caracterizou-se, mediante análise cinemática tridimensional baseada na videogrametria, o ângulo dorsal da articulação metacarpofalangiana de equinos em sete equinos da raça Puro Sangue Árabe. A análise tridimensional do movimento foi realizada em esteira rolante. O programa Dvideo foi utilizado para a obtenção das coordenadas tridimensionais do sistema de calibração e dos marcadores reflexivos posicionados na extremidade proximal do terceiro osso metacárpico, articulação metacarpofalangiana e extremidade distal da primeira falange. A articulação metacarpofalangiana apresentou extensão máxima durante o momento de apoio, no qual a face lateral do terceiro osso metacarpiano se apresentava de forma perpendicular ao solo. Foram observados dois picos de flexão durante a fase de elevação. Concluiu-se que a instrumentação utilizada para a análise cinemática tridimensional permitiu a investigação quantitativa da variação angular do movimento de extensão e flexão da articulação metacarpofalangiana de equinos por meio de imagens digitalizadas.
\end{abstract}

Palavras-chave: equino, ângulo articular, metacarpofalangiana, videogrametria

\begin{abstract}
The kinematic pattern of the metacarpophalangeal joint of equine using videogrammetry was evaluated. The three dimensional kinematic analysis were performed in a treadmill using seven Arabian horses. The Dvideo program was used to obtain the three dimensional coordinates from a calibration system and three reflective markers placed on the third metacarpus, metacarpophalangeal joint and proximal phalanx of the left forelimb. After the landing of the hoof, the fetlock extends to maximal loading at mid stance. During the swing phase the joint shows two flexion peaks. This method allows the determination of the flexion and extension angle of the metacarpophalangeal joint during locomotion.
\end{abstract}

Keywords: equine, articular angle, metacarpophalangeal, videogrammetry

\section{INTRODUÇÃO}

Os equinos são utilizados principalmente como atletas de alto desempenho. Animais expostos a exercícios que visam ao máximo rendimento são predispostos a desenvolver lesões por repetição e sobrecarga do sistema locomotor. Quando estas lesões acontecem, um dos sinais clínicos observados é a claudicação (Clayton et al., 2007). Durante a locomoção, a articulação metacarpofalangiana é submetida a estresses biomecânicos devido a sua composição anatômica permitir ampla gama de movimentos, especialmente durante competições de alta velocidade. A extensão durante a fase de apoio e a flexão na fase de suspensão causam forças de tensão nesta articulação e em seus tecidos de sustentação (Denoix et al., 1996). O alto grau de atividade torna esse conjunto particularmente suscetível a injúrias induzidas pelo exercício repetido (Bertone, 2004).

Recebido em 17 de março de 2010

Aceito em 28 de fevereiro de 2011

E-mail: raquel_albernaz@hotmail.com 
Os primeiros experimentos da análise do sistema locomotor de equinos foram desenvolvidos no século XIX. Em 1873 e 1894, Étienne Jules Marey, inventor e cronofotógrafo francês, estudou as características temporais como tempo de apoio e tempo de suspensão em diferentes andamentos, aplicando pela primeira vez métodos cinematográficos. Eadweard J. Muybridge, fotógrafo inglês, conhecido por seus experimentos com uso de múltiplas câmeras para captar movimento, em 1878, acionou câmeras fotográficas em determinada sequência para analisar o movimento do galope. Desde então, os avanços tecnológicos têm se desenvolvido rapidamente (Weeren, 2001; Weeren, 2002; Buchner, 2005). Considerando-se que as leis de Isaac Newton, publicadas em 1687, descrevem vários comportamentos relativos ao movimento de objetos e podem ser aplicadas ao movimento dos equinos, reconheceu-se que seu papel pode ser importante na etiologia, no diagnóstico e no tratamento das claudicações (Meershoek e Bogert, 2001).

Existem duas abordagens complementares para o estudo do corpo em movimento: análise cinética e cinemática. Para modificar a velocidade de um corpo em movimento, é preciso atuação de forças externas, as quais realizam certo trabalho. Este trabalho é uma apreciação da energia que o corpo apresenta pelo fato de estar em movimento em relação a um dado sistema de referência. A avaliação cinética é método pelo qual se estuda a determinante do movimento (Barrey, 1997). Esta análise pode ser realizada por meio de placa, ferradura ou pista de medição de força (Buchner, 2005).

A análise cinemática é o ramo da física que descreve os movimentos dos corpos no tempo e no espaço. Em equinos, pode ser utilizada para quantificar as características do andamento observadas durante o exame visual. Por meio desta ferramenta, é possível avaliar o deslocamento do segmento corporal assim como mensurar ângulos articulares em animais saudáveis ou claudicantes (Barrey, 1997; Bertone, 2003).

O sistema videográfico mais comum compreende câmeras de vídeo, sistema computacional e marcadores reflexivos aderidos à pele do animal (Bertone, 2003; Monina, 2007).O sistema tridimensional de coordenadas (X, Y, Z) é utilizado para definir a posição do membro no espaço (Wu e Cavanagh, 1995).

O entendimento do movimento da articulação metacarpofalangiana tem papel importante no diagnóstico e tratamento de lesões do sistema locomotor do equino. Portanto, os objetivos deste trabalho foram avaliar e descrever o método de análise cinemática tridimensional das características do movimento de flexão e extensão da articulação metacarpofalangiana de equinos caminhando em esteira rolante.

\section{MATERIAL E MÉTODOS}

A análise tridimensional da articulação metacarpofalangiana foi realizada no Laboratório de Fisiologia do Exercício de Equinos, Departamento de Morfologia e Fisiologia Animal, UNESP, Campus de Jaboticabal, SP. Foram utilizados sete animais clinicamente saudáveis da raça Puro Sangue Árabe, com média de idade de cinco anos, machos e fêmeas. Os equinos foram previamente adaptados à realização do exercício em esteira rolante (Esteira Galloper ${ }^{\circledR}$ Sahinco Ltda., 5500, Palmital, SP, Brasil) e submetidos a exame clínico geral e especial, com ênfase no sistema locomotor segundo Ross e Dyson (2005). Somente os animais hígidos foram considerados aptos a participarem desta pesquisa. O presente trabalho foi aprovado pelo Comitê de Ética e Bem-Estar da instituição, protocolo 028057-08.

Os animais selecionados após exame do sistema locomotor foram casqueados por profissional experiente da área. O ângulo da parede do casco de cada animal foi preservado. Todos os animais usaram ferraduras de metal e participaram do procedimento de filmagem na esteira rolante. Foram filmados em esteira rolante no andamento passo, velocidade de $1.4 \mathrm{~m} / \mathrm{s}$. A determinação da velocidade para filmagem foi realizada durante 0 procedimento de adaptação à esteira rolante, sendo observada a velocidade em que o animal mostrava-se confortável para realizar o exercício.

Para marcação das estruturas anatômicas referenciais, foram utilizados três marcadores esféricos reflexivos de $15 \mathrm{~mm}$ de diâmetro, apoiados sobre base de velcro fixada à pele do animal com auxílio de cola de secagem rápida para garantir sua permanência durante o movimento. As regiões anatômicas escolhidas 
para a colocação dos marcadores foram face proximal lateral esquerda do osso metacarpiano, face lateral esquerda da articulação metacarpofalangiana, face lateral esquerda distal da primeira falange. A pele dessas regiões foi previamente preparada por tricotomia, em uma área de aproximadamente $2 \mathrm{~cm}^{2}$, para a aderência do velcro.

Para o estabelecimento das regiões anatômicas, os animais foram posicionados em piso plano, com a finalidade de minimizar erros durante sua determinação. Segundo Eliashar et al. (2002), para se obter maior precisão, a marcação não deve ser feita sobre uma proeminência óssea específica, mas sobre o centro de rotação articular. As paredes do laboratório foram revestidas com tecido cor preto-fosca, para acentuar o contraste dos marcadores. Os animais foram filmados com auxílio de três câmeras de vídeo (Câmera filmadora Panasonic AG- DVC20 MiniDV), com seus respectivos tripés e iluminadores com lâmpada halógena (Lâmpada Halógena 127V 300W OSRAN) de 300W posicionados acima da objetiva de filmagem. As câmeras formavam ângulo de $45^{\circ}$ entre si, sendo duas orientadas com vista para a face lateral esquerda e a terceira câmera com vista frontal do membro anterior esquerdo do animal. As três câmeras foram posicionadas a aproximadamente $90 \mathrm{~cm}$ do solo.

Fitas de vídeo digitais (Fita MiniDV Panasonic 70,4m 60min LinearPlus) foram utilizadas para a gravação. As imagens capturadas foram exportadas para computador usando-se programa de captura de imagens AMCap (AMCap Microsoft Corporation) (capture application sample) e transformadas em arquivo de formato .avi.

Quatro fios de prumo (calibradores) fixados no teto do laboratório delimitaram a área de filmagem e a movimentação do membro anterior esquerdo. Ao longo deste fio de prumo, foram distribuídos seis marcadores esféricos reflexivos de $15 \mathrm{~mm}$ de diâmetro cada, distando $16 \mathrm{~cm}$ entre si. Na extremidade distal de cada calibrador, foi colocado um prumo de chumbo cônico pesando $450 \mathrm{~g}$. Cada calibrador foi numerado, assim como os seis marcadores presentes em cada um dos calibradores. A determinação das coordenadas dos marcadores foi realizada utilizando-se o método do nível da mangueira, fundamentado no princípio dos vasos comunicantes.

Para cada um dos 24 marcadores, foi determinado o valor de suas coordenadas no espaço em relação a um ponto zero, previamente delimitado, sendo $\mathrm{X}, \mathrm{Y}$ e $\mathrm{Z}$ equivalentes a comprimento, largura e altura, respectivamente. A gravação dos calibradores foi realizada para compor a etapa de calibração das câmeras, baseada no método Direct Linear Transformation elaborado por Abdel-Aziz e Karara (1971), dando origem ao arquivo .cal.

O corte, a sincronização e o rastreamento das imagens foram realizados com o auxílio do software Dvideo (Digital video for Windows, version 5.0) desenvolvido na Faculdade de Educação Física da UNICAMP. A partir dos arquivos.avi., as imagens foram segmentadas no intervalo de interesse usando-se frequência de 60 frames por segundo. Analisou-se a sequência de seis passadas completas, sincronizadas entre as câmeras com o uso da claquete. Os momentos escolhidos para análise foram impacto, suporte e final do tempo decolagem do passo. A fase de impacto compreendeu o momento imediatamente antes de o casco entrar em contato com o solo; a fase de suporte foi definida quando o peso do animal foi aplicado ao membro, no instante em que o terceiro osso metacarpiano formava $90^{\circ}$ com o solo. O final do momento de decolagem compreendeu o último toque da pinça ao solo antes da fase de elevação (Back, 2001; Parks, 2003; Clayton, 2004; Thomason e Peterson, 2008).

O rastreamento das imagens foi realizado na frequência de 30 frames por segundo e salvo no formato .dat. A partir dos arquivos .avi., .dat. e .cal., foi possível realizar a reconstrução tridimensional das imagens. Após este procedimento, o programa de análise do movimento utilizado (Dvideo) oferece um arquivo de dados contendo as coordenadas espaciais dos marcadores medidos em cada campo. Após a realização da reconstrução tridimensional, em ambiente Matlab (Matrix Laboratory), aplicaram-se funções matemáticas para a obtenção dos valores angulares da articulação metacarpofalangiana.

Com o objetivo de avaliar a precisão do arquivo de referência do laboratório, foi realizado o 
“teste do bastão", ou teste de medidas conhecidas. Após o posicionamento das câmeras, conforme descrito anteriormente, realizou-se, durante 10 segundos, a filmagem do sistema de calibração com os calibradores dispostos em seus respectivos lugares. Todo o procedimento de filmagem foi realizado com o uso do controle remoto para evitar qualquer tipo de movimentação das câmeras. Os calibradores foram retirados, e foi gravado um membro da equipe percorrendo o espaço delimitado pelos calibradores portando um bastão de $439 \mathrm{~mm}$ de comprimento. Esse bastão possuía em suas extremidades dois marcadores esféricos reflexivos. Posteriormente, as imagens obtidas foram transferidas para o computador, sincronizadas, cortadas e rastreadas.
A distância entre os marcadores calculada em movimento, por meio da videogrametria, foi de 438mm. Comparada com o tamanho real do bastão, 439mm, obteve-se o desvio-padrão de $5,05 \mathrm{~mm}$. Os dados foram submetidos aos testes de pressuposições para normalidade dos erros (Teste de Kolmogov Smirnov) e homogeneidade das variâncias (homocedasticidade) (Teste de Levème), com nível de significância estipulado em $5 \%$.

\section{RESULTADOS E DISCUSSÃO}

Os resultados obtidos no presente estudo estão apresentados na Tab. 1 e na Fig. 1. A análise cinemática tridimensional, realizada com 0 auxílio da videogrametria, permitiu a investigação quantitativa do ângulo da AMF por meio de imagens digitalizadas.

Tabela 1. Ângulo da articulação metacarpofalangiana de equinos da raça Puro Sangue Árabe, de acordo com os momentos analisados

\begin{tabular}{cccccccc} 
Momento & \multicolumn{7}{c}{ Animal } \\
& 1 & 2 & 3 & 4 & 5 & 6 & 7 \\
\hline Impacto & 172.64 & 168.90 & 170.15 & 169.36 & 163.09 & 161.16 & 168.32 \\
Suporte & 138.58 & 128.49 & 134.51 & 140.26 & 126.63 & 127.50 & 126.56 \\
Decolagem & 164.18 & 155.11 & 156.64 & 159.18 & 153.66 & 142.76 & 154.11 \\
\hline
\end{tabular}

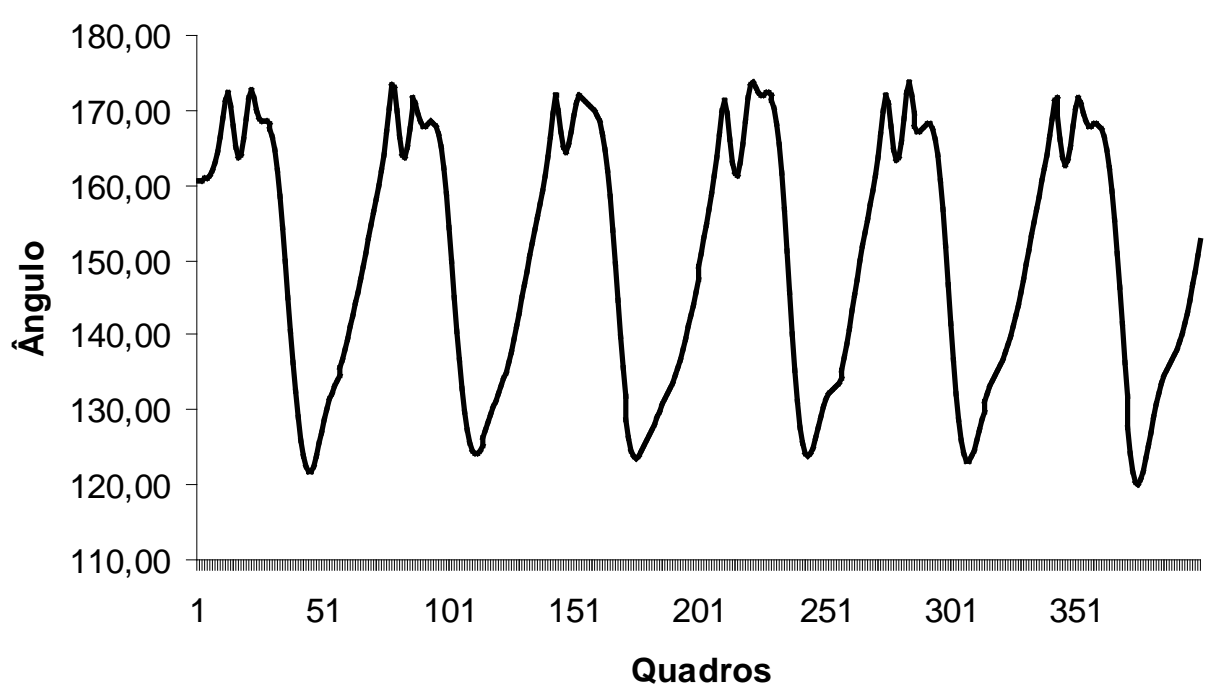

Figura 1. Representação gráfica da variação angular do movimento da articulação metacarpofalangiana de equinos da raça Puro Sangue Árabe em relação ao número de quadros.

A utilização da esteira rolante, que oferece o controle da velocidade, assim como das condições ambientais, garantiu maior uniformidade e padronização do movimento. Segundo Bertone (2003), a esteira rolante é uma ferramenta útil para tal análise em condições controladas a velocidades constantes. A esteira rolante permite ainda a gravação das passadas ao longo do tempo, sem que haja movimento da câmera de gravação. Para melhor confiabilidade 
dos dados, realizou-se o teste de aferição da precisão de medidas, ou teste do bastão, por meio do qual foi possível conferir o sistema de calibração e das coordenadas espaciais do movimento. Neste estudo, obteve-se desviopadrão de 5,05mm. Em estudo realizado em condições semelhantes (Christovão, 2006), o desvio-padrão foi igual a $3,5 \mathrm{~mm}$, porém o número de quadros analisados foi 10 vezes menor.

A análise do movimento foi realizada apenas ao passo devido ao fato de a proximidade dos marcadores impossibilitar o rastreamento em velocidades maiores nas condições deste experimento. Porém, segundo Back (2001), a análise cinemática realizada ao passo é equivalente e apresenta correlação com a análise ao trote, portanto pode-se predizer a cinemática do trote com base nos achados ao passo.

O movimento angular das articulações pode ser descrito em três direções: flexão e extensão; adução e abdução; e rotação interna e externa, porém o membro dos equinos apresenta capacidade restrita em executar movimentos de rotação interna ou externa (Clayton, 2004). Neste experimento, foi analisado o movimento angular de flexão e extensão. Chateu et al. (2004) não observaram efeito de elevação do talão sobre o movimento de adução e abdução ou rotação interna e externa da articulação metacarpofalangiana, confirmando que o movimento angular desta articulação é caracterizado principalmente pela flexão e extensão do membro.

A opção da colocação de marcadores sobre a pele pode sofrer influência da movimentação desta sobre os ossos dos segmentos, e, por isso, foram escolhidos pontos onde essa interferência fosse minimizada. Segundo Back (2001), não são necessários corretores para o deslocamento de pele na análise angular da articulação metacarpofalangiana.

A articulação metacarpofalangiana apresentou extensão máxima durante o momento de apoio, no qual a face lateral do terceiro osso metacarpiano se apresentava de forma perpendicular ao solo. Foram observados dois picos de flexão durante a fase de elevação, sendo o primeiro após o momento de contato inicial do casco com o solo, onde o ângulo da articulação metacarpofalangiana tendeu a formar um platô, e o segundo momento após a retirada do membro do solo (Fig. 1).

\section{CONCLUSÕES}

O método de análise cinemática tridimensional, realizado com o auxílio da videogrametria, possibilitou a aquisição de dados analíticos confiáveis e reproduzíveis. A partir deste método, foi possível executar a investigação quantitativa da variação angular da articulação metacarpofalangiana por meio de imagens digitalizadas, contribuindo, assim, para o estudo do sistema locomotor dos equinos.

\section{AGRADECIMENTOS}

Professor Ricardo Leite de Barros, pelo auxílio nas análises computacionais e pelo programa Dvideo. À CAPES, pela bolsa de doutorado concedida para este estudo.

\section{REFERÊNCIAS BIBLIOGRÁFICAS}

ABDEL-AZIZ, Y.I.; KARARA, H.M. Direct linear transformation from comparator coordinates into object-space coordinates in close-range photogrammetry. In: CLOSERANGE PHOTOGAMMETRY, 1971, Falls Church, VA. Proceedings... Falls Church: American Society of Photogrammetry, 1971. p.118.

BACK, W. The role of the hoof and shoeing. In: BACK, W.; CLAYTON, H. Equine locomotion. London: W B Saunders, 2001. cap.6, p.135-166.

BARREY, E. Locomotion variables. In: BARREY, E.; FAZIO, E.; FERLAZZO, A. et al. Performance diagnosis of horses. Netherlands: Wageningen Pears, 1997. cap.5, p.72-96.

BERTONE, A.L. Gait analysis for the quantification of lameness. In: ROSS, M.W.; DYSON, S. Diagnosis and management of lameness in the horse. St Louis, Mo: Saunders, 2003. p. 222-225.

BERTONE, A.L. Distal Limb: fetlock and pastern. In: HINCHCLIFF, K.; KANEPS, A.; GEOR, R.J. Equine sports medicine and surgery: basic and clinical sciences of equine athlete. St Louis, Mo: Saunders, 2004. cap. 16. 
BUCHNER, H.H.F. Distal limb internal dynamics: joint moments, tendon forces, and lessons for orthopedic shoeing. In: ANNUAL CONVENTION OF THE AMERICAN ASSOCIATION OF EQUINE PRACTITIONERS, 51., 2005, Seattle, Washington. Proceedings... Seattle: AAEP, 2005. p.134-140.

CHATEAU, H.; DEGUEURCE, C.; DENOIX, J.M. Effects of $6^{\circ}$ elevation of the heels on 3D kinematics of the distal portion of the forelimb in the walking horse. Equine Vet. J., v.36, p.649654, 2004.

CHRISTOVÃO, F.G. Análise cinemática tridimensional do movimento de equinos em esteira rolante. 2006. 108f. Tese (Doutorado em Medicina Veterinária) - Faculdade de Ciências Agrárias e Veterinárias, Universidade Estadual Paulista, Jaboticabal, SP.

CLAYTON, H. The dynamic horse: a biomechanical guide to equine movement and performance. Mason: Sport Horse Publication, 2004

CLAYTON, H.M.; SHA, D.; STICK, J. et al. 3D Kinematics of the Metacarpophalangeal joint at walk and trot. Vet. Comp. Orthop. Traumatol.,v.20, p.86-91, 2007.

DENOIX, J.M.; STEPHANIE, J.B.; BOUSSEAU, B. et al. Ultrasonographic anatomy of the dorsal and abaxial aspects on the equine fetlock. Equine Vet. J., v.28, p.54-62, 1996.

ELIASHAR, E.; GUIGAN, M.P.; ROGERS, K.A. et al. A comparison of three horseshoeing styles on the kinetics of breakover in sound horses. Equine Vet. J., v.34, p.184-190, 2002.
MEERSHOEK, L.S.; BOGERT, A.J. Mechanical analysis of locomotion. In: BACK, W.; CLAYTON, H. Equine locomotion. London : W B Saunders, 2001. cap.13, p.305-326.

MONINA, M.I. Biomecânica equina. In: BOFFI, F.M. Fisiologia del ejercicio en equinos. Buenos Aires: Inter-Médica, 2007. cap. 14, p.177-203.

PARKS, A. The foot and shoeing. In: ROSS, M.W.; DYSON, S. Diagnosis and management of lameness in the horse. St Louis, Mo: Saunders, 2003. p.250-275.

ROSS, M.W.; DYSON, S.J. Diagnosis and management of lameness in the horse. St Louis, Mo: Saunders, 2005. p.348-362.

THOMASON, J.J.; PETERSON, M.L. Biomechanical and mechanical investigation of the hoof-track interface in racing horses. Vet. Clin.: Equine Pract., v.24, p.53-77, 2008.

WEEREN, P.R. The clinical applicability of automated gait analysis systems. Equine Vet. J., v.34, p.218-219, 2002.

WEEREN, P.R. History of locomotor research. In: BACK, W.; CLAYTON, H. Equine locomotion. London: W B Saunders, 2001. cap.1, p.1-35.

WU, G.; CAVANAGH, P.R. ISB recommendations for standardization in the reporting of kinematic data. J. Biomech., v.28, p.1257-1261, 1995. 